\title{
Ownership vs. Stewardship
}

I HAVE BEEN THINKING recently about the complementary roles of stewardship and ownership in the context of the Internet. A significant fraction of the physical infrastructure of the Internet and the equipment that animates the World Wide Web is privately owned. Some of these components are "owned" by governments and in some cases by cooperatives. The owners are usually motivated by the benefits of their ownership whether that is making a profit, fulfilling government obligations, or producing benefit for the group owners in the case of a cooperative. There are, of course, cases in which profit is not a motive but rather social benefit. Think of schools, churches, and libraries that provide access to the Internet freely. They are the owners of the facilities and operate them in part to fulfill their missions. So where does stewardship fit into this picture?

Organizations such as the Regional Internet Registries ${ }^{\mathrm{a}}$ (ARIN, RIPE, LACNIC, AFRINIC and APNIC) and the Internet Corporation for Assigned Names and Numbers ${ }^{\mathrm{b}}$ (ICANN) do not own the assets they administer. Their missions are to manage the assignment of these assets to parties who have the right to use these assets in exchange for fees paid to maintain these assignments. In the case of domain names, the registrars are often, but not always, for-profit entities that assist users to register and maintain a record of the assignment. The domain name registries may also be for-profit, non-profit, or even government operated in the case of the country-code Top Level Domains.

\footnotetext{
a https://en.wikipedia.org/wiki/Regional_Internet_registry

b https://www.icann.org/
}

Interestingly, in the case of domain names, the assets (that is, right to use) is created by the registrant who can invent new names not previously registered and register them for use. In the case of Internet address space, there is a finite amount of that space (IPv4 has 32 bits of address space, IPv6 has 128-a lot!) and the right to use is meted out by ICANN to the Regional Internet Registries, which is in turn typically meted out to Internet service providers who temporarily or even dynamically allocate addresses to end users for the purpose of their gaining access to the Internet and its many services.

The Regional Internet Registries and ICANN are stewards of IP address and domain name spaces. Their role is, to the best of their abilities, to provide fair access to these assets and to keep track of these assignments to ensure parties who do not have a registered right to use them cannot falsely claim or hijack them. While opinions may vary as to the success of these institutions in carrying out their missions, it strikes me as very interesting that the largely private sector ownership of the physical Internet (including the hosts and cloud datacenters) is ultimately dependent on the successful stewardship of a few key non-profit entities for the useful application of these physical assets. The Web would not exist in its present form without domain names. Domain names would not be useful if they could not be mapped into IP addresses so the servers of those domain names could be reached on the Internet.

Given the understandable economic motivations of for-profit institutions, the fact that the Internet and the World Wide Web are dependent on the stewardship of a key set of non-profit institutions strikes me as quite remarkable. It might easily have gone the other way. There is a current trend toward encouraging technology transfer from government-sponsored research into the private sector. I agree with the general premise that publicly funded research should find its way into the private sector where investment and hard work can produce economic gains, jobs, and useful products and services. It is nonetheless fascinating to me that one of the largest government-produced engines of economic growth and innovation is so deeply dependent on the stewardship of the people and institutions that administer Internet addresses and domain names.

The Internet Society (ISOC) is another non-profit institution, which houses the Internet Architecture Board $^{\mathrm{d}}$ (IAB), the Internet Engineering Task Force (IETF), and the Internet Research Task Force ${ }^{f}$ (IRTF). ISOC benefits directly from its wholly owned, non-profit subsidiary, the Public Interest Registry ${ }^{g}$ (PIR), which is the steward of the .org top level domain. The Internet Society plays an active role in reminding us of the importance of stewardship and the need to heighten awareness that harmful uses of the Internet threaten its global connectedness and the safety of its users. We are all in debt to the stewards of the Internet. Long may they serve.

\section{c http://www.isoc.org \\ d http://www.iab.org \\ e http://www.ietf.org \\ f http://www.irtf.org g http://www.pir.org}

Vinton G. Cerf is vice president and Chief Internet Evangelist at Google. He served as ACM president from 2012-2014.

Copyright held by author. 